\section{Reactive arthritis induced by recurrent $C$. difficile colitis}

\author{
Allison Marr, ${ }^{1}$ Ryan Mertens, ${ }^{1}$ \\ Darla Klug Eastman² \\ 'Iowa Methodist Medical Center; \\ 'Critical Care Veterans' Affairs Central \\ lowa Healthcare System Drake University \\ College of Pharmacy and Health Sciences \\ Des Moines, IA, USA
}

\begin{abstract}
Clostridium difficile colitis is a common infection that can be difficult to resolve and may result in recurrent infections. Reactive arthritis is a rare presentation of this disease and its treatment is not well differentiated in the literature. We describe a case of reactive arthritis occurring in a patient with a history of recurrent Clostridium difficile colitis while currently receiving a taper of oral vancomycin. His arthritis symptoms resolved with corticosteroids and continued treatment with anticlostridial antibiotics.
\end{abstract}

\section{Introduction}

Clostridium difficile colitis is becoming more common and can be difficult to cure. Once $C$. difficile infection has occurred, risk for re-infection and subsequent recurrences are increased. Reactive arthritis is a rare, but recognized, reaction in patients with $C$. difficile colitis. We report a case of a patient who developed reactive arthritis after a year of recurrent episodes of $C$. difficile colitis.

\section{Case Report}

A 68-year-old male, weighing $112 \mathrm{~kg}$, was admitted for a ten day history of polyarthritis with associated joint swelling. Leading up to admission, the patient had a history of recurrent $C$. difficile colitis, secondary to extensive diverticular disease which had been treated with multiple rounds of antibiotics.

His long history of recurrent $C$. difficile colitis started almost a year earlier. In October 2009, he was first admitted for diverticulitis and treated with three separate rounds of antibiotics for complicated diverticular disease. Within the following few months, he developed persistent diarrhea and was twice hospitalized in November and December 2009 for confirmed $C$. difficile toxin positive colitis and treated with metronidazole initially and then switched to vancomycin. Following these hospitalizations, he completed a vancomycin taper over two months and his colitis was managed on lactobacillus therapy for most of the next year. A colonoscopy in February 2010 showed diverticular disease of the sigmoid colon with reddened mucosa; however, biopsies were negative for neoplasm or pseudomembranes at that time. More recently, a month before admission, he was found to have $C$. difficile toxin positive stools and resumed oral vancomycin therapy that he continued up until hospitalization. Approximately, three weeks after resuming oral vancomycin therapy, he developed malaise, myalgias, and arthralgias in multiple joints. The day prior to admission, the patient presented to his primary care physician with these worsening complaints.

The patient's past medical history was significant for recurrent $C$. difficile infections and diverticulitis, coronary artery disease, hyperlipidemia, depression, obstructive sleep apnea, and multiple TIAs in the late 1990's. His home medications upon admission included aspirin, simvastatin, venlafaxine, and a vancomycin taper.

Examination of the patient revealed an afebrile man with noticeable swelling and erythema of the left wrist and elbow, as well as some minimal swelling involving his left forearm. The joints were warm to the touch, tender to palpation, and painful with movement to the point of severely restricting normal range of motion. His knees were tender to palpation bilaterally but free of effusions, erythema, and swelling. He had a moderate left periorbital edema that was erythematous and warm to the touch when compared to the right. The patient denied any vision changes associated with his left eye. He had been using high doses of naproxen and acetaminophen to help with his pain but reported minimal relief. He did have some mild arthritis in his knees but denied any additional problems with joint pain previously. Excluding his ongoing $C$. difficile colitis, he denied any recent illnesses, trauma, dysuria, urethral discharge, conjunctivitis, fevers, or chills.

Laboratory studies on admission showed a white blood cell count of $17,700 / \mathrm{mm}^{3}$, hemoglobin of $14.8 \mathrm{~g} / \mathrm{dL}$, ESR of $72 \mathrm{~mm} / \mathrm{hr}$, CRP of 15.9 $\mathrm{mg} / \mathrm{dL}$, rheumatoid factor of less than 20 IU/mL, two negative blood cultures, and a normal uric acid and CPK. There were no other cultures obtained since the patient did not have any urinary or pulmonary symptoms. Serology for Parvovirus, Lyme's disease, and West Nile virus were all negative. Abdominal CT obtained in the office prior to admission revealed inflammation in the distal descending colon and proximal sigmoid colon. Stools were positive for fecal lactoferrin and $C$. diffi-
Correspondence: Darla Klug Eastman, 106 Cline Hall, 2507 University Avenue, Des Moines, IA 50311, USA. Tel. +1.515.699.5999 ext 4057 .

Fax: +1.515.699.5672.

E-mail: darla.eastman@drake.edu

Key words: reactive arthritis, Clostridium difficile colitis, recurrence.

Contributions: AM, performed background research and writing of drafts and editing of case report; RM, house physician involved in patient care, writing of patient case; DKE, writing of drafts and editing process of case report.

Conflict of interest: the authors report no conflicts of interest.

Received for publication:6 June 2011.

Revision received: 12 August 2011.

Accepted for publication: 25 November 2011.

This work is licensed under a Creative Commons Attribution NonCommercial 3.0 License (CC BYNC 3.0).

(C) Copyright A. Marr et al., 2012

Licensee PAGEPress, Italy

Rheumatology Reports 2012; 4:e1

doi:10.4081/rr.2012.e1

cile toxin and negative for Salmonella, Shigella, Camphylobacter, and Shiga toxin 1 and 2. Of note, the specific strain of $C$. difficile toxin was not specified by the laboratory. Arthrocentesis of the left elbow revealed a WBC count of $50,000 / \mathrm{mm}^{3}$ with $15 \%$ neutrophils and $68 \%$ mononuclear cells. Crystal examination, gram stain, and cultures were negative for the joint aspirate. X-rays of the left wrist and forearm were negative for any abnormalities.

Once septic arthritis was ruled out with benign synovial fluid analysis, the patient was started on a prednisone burst and taper beginning with $30 \mathrm{mg}$ daily. This was preceded with a one-time dose of $30 \mathrm{~g}$ of intravenous immunoglobulin for his $C$. difficile colitis per the infectious disease physician on the case. The day following his initial prednisone dose he reported resolution of his joint pains and stiffness. He had a noticeable decrease in the swelling around his left elbow, wrist, and forearm. He also had a return of full range of motion in all joints affected. His WBC count came down to $12,200 / \mathrm{mm} 3$ and he reported feeling much better.

He was discharged home on a prednisone taper as well as a vancomycin taper. He reported having semi-formed stools that were mucous free, no abdominal pain or overt symptoms of colitis.

After discharge, some arthritis symptoms persisted and he required an increased dose of 
corticosteroids. He received prednisone $30 \mathrm{mg}$ daily for four weeks and then was gradually tapered down to zero, as improvements were seen in patient-reported symptoms and inflammatory markers. One month after discharge, his ESR had decreased to $9 \mathrm{~mm} / \mathrm{hr}$ and his CRP had dropped to $1.6 \mathrm{mg} / \mathrm{dL}$. The most recent follow-up that this patient had with his primary care physician was 4 months after admission and at that time his labs showed a negative $C$. difficile toxin assay and positive fecal lactoferrin. He did not have any other labs at this time and was currently off his anticlostridial medications.

\section{Discussion}

We present a case of reactive arthritis that developed after multiple recurrences of $C$. difficile colitis. $C$. difficile colitis is most commonly associated with persistent diarrhea; however, various extra colonic manifestations, in which diagnosis can be difficult, has been documented in a small number of patients. ${ }^{1}$ The diagnosis of $C$. difficile reactive arthritis was first documented over 20 years ago, but the authors found no documented cases of reactive arthritis in a patient with recurrent $C$. difficile infections. The 2010 Clinical Practice Guidelines for $C$. difficile comment on the rare but possible occurrence of arthritic manifestations of disease, but do not elaborate on diagnostic criteria or provide pharmacotherapy recommendations. ${ }^{2}$ Due to the limited number of reported cases, the pathophysiology is not well studied and there are no recommended therapies. ${ }^{3}$ There is still little known about the diagnosis and treatment of $C$. difficile associated reactive arthritis aside from a few published case reports and author hypotheses.

An article by Putterman et al. in 1993 proposed criteria for the diagnosis of $C$. difficile reactive arthritis. These criteria include the presence of four cardinal features: i) appearance of arthritis together with or following the onset of diarrhea and/or colitis; ii) diarrhea appearing some time after a course of systemic antimicrobial treatment; iii) microbiologic proof of $C$. difficile involvement (either positive stool culture or assay for toxin); iv) no reasonable alternative diagnosis for the arthritis or diarrhea (no other identified infectious agents). ${ }^{4}$

Based on the aforementioned criteria, we believe that our patient developed $C$. difficile reactive arthritis. Infectious arthritis was ruled out since there was a negative culture and gram stain on arthrocentesis. Another common cause of polyarthritis, psoriatic arthritis, was considered less probable since there is no diagnosis of psoriasis. The patient's recent colonoscopy showed diverticu- lar disease of the sigmoid colon but there was no sign of inflammatory bowel disease, including Crohn's disease or ulcerative colitis, which can also cause reactive arthritis. No other cause of infection or arthritis was identified including rheumatoid arthritis, gout, West Nile virus, and Parvovirus.

Putterman et al. provided an initial review of the published literature by analyzing 17 patients, finding that arthritis followed diarrhea in an average of 8-35 days, and large joints were more commonly affected than smaller joints. Laboratory values reported in this review support our findings, which include an elevated ESR and peripheral white blood cell count, and aspirated joint fluid with elevated WBC but negative cultures and without fluid crystals. Rheumatoid factors were found to be negative, as was the case in our patient. Although most patients in the literature presented febrile upon admission, our patient was afebrile, but this may be explained by his self-dosing of naproxen and acetaminophen. ${ }^{4}$

Another review compiled by Jacob et al. in 2001 compared the disease history in 36 patients. Our case patient has many similarities to these case patients with documented $C$. difficile reactive arthritis, including onset of occurrence of joint symptoms within a month after diarrhea began, elevated ESR and treatment with concomitant anti-inflammatory plus anticlostridial medication. However, none of these cases were noted to have occurred in patients who had experienced multiple recurrent episodes of $C$. difficile making this case novel and of importance for clinicians. ${ }^{1}$ Since this review, there have been only a handful of additional case reports published that document $C$. difficile reactive arthritis and still none with recurrent cases of colitis.

Many authors in past literature reviews and case studies have established an increased prevalence of HLA-B27 in their reactive arthritis patients. However, a recent article published by Townes et al stated that past authors may have overestimated the contribution of HLA-B27 to the risk of reactive arthritis. ${ }^{5}$ We chose not to draw an HLA-B27 marker for our case patient during his hospital admission. We believed that we had sufficient evidence for the diagnosis of $C$. difficile reactive arthritis based on clinical presentation and other laboratory markers listed previously and this laboratory result would not have impacted the patient's care.

Similarly, we did not check for chlamydia, another known cause of reactive arthritis, since our patient did not specifically exhibit risk factors or signs and symptoms of this disease. $^{3}$

Our lab does not test for different toxin strains of $C$. difficile, so it is unknown if our patient had the same strain throughout his many recurrences, as his reactive arthritis symptoms did not appear until approximately 8 months after his initial $C$. difficile toxin positive stool. If in fact, it was a different strain, then it could be possible that the immune system responded differently during this recurrence then in the past as the immune system may have come into contact with new antigens.

There is not a recommended treatment regimen for $C$. difficile reactive arthritis; however, review of the literature reveals that many physicians treat with NSAIDs and corticosteroids (both systemic and intra-articular), in addition to anticlostridial medications, such as metronidazole, oral vancomycin and other adjunctive therapies including cholestyramine and rifaximin.,6-8 Jacobs et al. states that rheumatologists have utilized various treatment modalities; including, joint fluid drainage, intraarticular steroid administration and nonsteroidal anti-inflammatory therapies without concluding a superior treatment modality. A study by Kocar et al. concluded treatment of arthritis may resolve with anticlostridial treatment alone in fifty percent of patients. ${ }^{1}$ This is the only study, to our knowledge, that concluded sole treatment with anticlostridial antibiotics is effective at resolving reactive arthritis symptoms caused by $C$. difficile. ${ }^{7}$ Our case provides significant evidence that patients may require therapy beyond anticlostridial antibiotics since this patient was already being treated with oral vancomycin at the onset of symptoms. Despite ongoing treatment with vancomycin, the patient's symptoms worsened until corticosteroids were started and resulted in dramatic improvement.

One difference in this case compared with other published case reports is that our patient was treated with intravenous immunoglobulin (Flebogamma), in addition to oral corticosteroids and oral vancomycin. Intravenous immunoglobulin is thought to help the body to mount an immune response to $C$. difficile and the associated toxins to prevent recurrent episodes of infection. ${ }^{9}$ A review article published in the NEJM does discuss the possibility of immunotherapy being beneficial; even though there is currently no data available from randomized controlled trials. The article recommends a dose of $400 \mathrm{mg} / \mathrm{kg}$ of immunoglobulin, which is less then this case patient received. ${ }^{10}$ The use of intravenous immunoglobulin in our case patient was utilized as adjunctive therapy to treat his $C$. difficile in the hopes of preventing future recurrent episodes that may precipitate another case of reactive arthritis. There is no evidence to support the use of intravenous immunoglobulin for treatment of reactive arthritis and we cannot speculate on what role, if any, it played in this case.

There is little documented follow-up data in 
adult patients with reactive arthritis due to $C$. difficile infections, so it is unknown if the reactive arthritis symptoms recur in the future. Putterman et al provided follow-up for six of the 17 case reports; one patient had pain requiring NSAID use over a two year period while all others remained asymptomatic. ${ }^{4}$ In contrast, a case report by Cope et al., published in 1992, described a case of arthritic symptoms that persisted for several years after the initial episode of arthritis following a diagnosis of $C$. difficile. ${ }^{11}$ Since this was our patient's first incidence of reactive arthritic symptoms, we are unable to further comment on symptom recurrence; however, additional research into this could provide valuable insight to providers in the future.

There is also little known data on the duration of arthritic symptoms. Jacobs et al found data in their case reports documenting arthritis symptoms lasting from two days to almost one year. ${ }^{1}$ However, as in all forms of reactive processes, it is imperative to control the underlying problem, which, in this case, is the $C$. difficile colitis. In the future, it may be helpful to evaluate the clinical status of these patients multiple years post-diagnosis to access recurrence rates and define clinical endpoints.

\section{Conclusions}

We present a case of $C$. difficile reactive arthritis occurring despite therapy with oral vancomycin following multiple recurrent bouts of $C$. difficile colitis. Our patient's recurrent nature of $C$. difficile is unique to the available literature on $C$. difficile reactive arthritis. Furthermore, this case provides evidence that both anticlostridial antibiotics and corticosteroids may be required to improve clinical condition. Our patient had received several months of vancomycin therapy yet still developed a case of $C$. difficile reactive arthritis. $C$. difficile is a rare cause of reactive arthritis but it is important that clinicians are aware of this association when treating an increasing number of patients with a history of $C$. difficile infection so that cases may be properly diagnosed and treated, thereby avoiding delays in therapy and unnecessary, expensive diagnostic tests.

\section{References}

1. Jacobs A, Barnard K, Fishel R, Gradon JD. Extracolonic manifestations of Clostridium difficile infections. Medicine 2001;80:88-101.

2. Cohen SH, Gerding DN, Johnson S, et al. Society for Healthcare Epidemiology of America; Infectious Diseases Society of America: Clinical practice guidelines for Clostridium difficile infection in adults: 2010 update by the Society for Healthcare Epidemiology of America (SHEA) and the Infectious Diseases Society of America
(IDSA). Infect Control Hosp Epidemiol 2010;31.

3. Carter JD, Hudson AP. Reactive arthritis: Clinical aspects and medical management. Rheum Dis Clin N Am 2009;35:21-44.

4. Putterman C, Rubinow A. Reactive arthritis associated with Clostridium difficile pseudo-membranous colitis. Semin Arthritis Rheum 1993;22:420-6.

5. Townes JM. Reactive arthritis after enteric infections in the United States: The problem of definition. Clin Inf Dis 2010;50:24754.

6. Birnbaum J, Bartlett JG, Gelber AC. Clostridium difficile: an under-recognized cause of reactive arthritis? Clin Rheumatol 2008;27:253-5.

7. Kocar IH, Caliskaner Z, Pay S, Turan M. Clostridium difficile infection in patients with reactive arthritis of unknown etiology. Scand J Rheumatol 1998;27:357-62.

8. Bartlett JG. New antimicrobial agents for patients with Clostridium difficile infections. Curr Infect Dis Rep 2009;11:21-8.

9. Abougergi MS, Kwon JH. Intravenous immunoglobulin for the treatment of Clostridium difficile infection: A review. Dig Dis Sci 2011;56:19-26.

10. Kelly CP, LaMont JT. Clostridium difficile More difficult than ever. N Engl J Med 2008;359:1932-40.

11. Cope A, Anderson J, Wilkens E. Clostridium difficile toxin induced reactive arthritis in a patient with chronic Reiter's syndrome. Eur J Clin Microbiol Infect Dis 1992;11:40-3. 